\title{
Microallelotyping Defines the Monoclonal or the Polyclonal Origin of Mixed and Collision Endocrine-Exocrine Tumors of the Gut
}

\author{
Daniela Furlan, Roberta Cerutti, Anna Genasetti, Giuseppe Pelosi, Silvia Uccella, \\ Stefano La Rosa, and Carlo Capella
}

Department of Clinical and Biological Sciences (DF, RC, AG, SU, CC), University of Insubria, Varese, Department of Pathology and Laboratory Medicine (GP), European Institute of Oncology, Milan, and Department of Pathology (SL), Hospital Fondazione Macchi, Varese, Italy

SUMMARY: Mixed endocrine-exocrine tumors of the gut are a heterogeneous group of neoplasms with uncertain histogenesis showing different morphologic and clinical features. The aim of this work is to clarify the histogenesis of these tumors by studying the genetic profile of both the endocrine and exocrine components. We performed an allelotyping analysis of five mixed endocrine-exocrine tumors (two gastric and three colonic) and one rectal collision tumor, using 35 polymorphic microsatellite markers covering a total of six chromosomes, including 3, 5q, 6, 11, 17, and 18. The loss of heterozygosity (LOH) analysis showed concurrent losses of the same allele in both the endocrine and exocrine components in all of the five mixed tumors composed by a poorly differentiated endocrine carcinoma or a well differentiated endocrine carcinoma associated with adenocarcinoma or adenoma. Among these tumors an identical LOH pattern was frequently found on chromosomes 17p, 18q, and $5 \mathrm{q}$. Additional allelic losses restricted to the poorly differentiated endocrine carcinoma were often observed. On the contrary, in the only collision tumor composed by a well differentiated endocrine carcinoma associated with adenocarcinoma, completely different allelotypes between the two components were detected. These findings confirm a close genetic relationship between the two distinct histologic components within mixed endocrine-exocrine tumors, supporting the hypothesis that a monoclonal mechanism of tumorigenesis is the most frequent genetic event in mixed exocrine-endocrine tumors. The clonal divergence observed in the only collision tumor, composed by a well differentiated endocrine carcinoma associated with an adenocarcinoma, confirms the existence of double tumors growing next to each other coincidentally but showing different histogenesis and different tumorigenetic pathways. (Lab Invest 2003, 83:963-971).

$M$ ixed endocrine-exocrine tumors of the gut constitute a heterogeneous group of rare neoplasms, which includes different histopathologic and prognostic classes. Microscopically, they consist of an adenomatous and/or carcinomatous component associated either with a well differentiated endocrine tumor (WDET) or with a poorly differentiated endocrine carcinoma (PDEC). The endocrine component represents at least one-third to half of the tumor tissue, either intimately and diffusely admixed with the exocrine component (combined tumors) or occurring mainly as separate areas in the same neoplasm (composite tumors) (Capella et al, 2000). Endocrineexocrine collision tumors are composed of two topographically clearly separated components and by definition are not included in the category of mixed tumors.

\section{DOI: 10.1097/01.LAB.0000079006.91414.BE}

Received December 13, 2002.

This study was in part supported by a grant from the University of Insubria, Varese, Italy.

Address reprint requests to: Dr. Carlo Capella, Department of Pathology, Ospedale di Circolo, Viale Borri 57, 21100 Varese, Italy. E-mail: carlo.capella@ospedale.varese.it
According to such morphologic classification, two opposite hypotheses have been proposed regarding the histogenesis of these biphenotypic tumors. One model suggests that the exocrine and endocrine components expand from two distinct precursors through different tumorigenetic pathways resulting in double tumors (named "collision-type tumors") that exist next to each other coincidentally. In this view, the endocrine tumor with adjacent satellite adenocarcinoma was suggested to exert paracrine effects on the surrounding glandular epithelial cells (Peison and Benisch, 1983; Yamashita and Flinner, 1985). However, there are no molecular studies supporting this model.

The other hypothesis suggests a monoclonal origin of the two components from a pluripotent epithelial stem cell undergoing an anaplastic transformation with a bidirectional differentiation. In agreement with this model, two different studies have recently reported a microallelotyping analysis of seven colorectal PDECs (Vortmeyer et al, 1997) and of eight gastric PDECs (Kim et al, 2002) associated with adenomas or adenocarcinomas. In both studies, using a genomewide approach (Kim et al, 2002) or a loss of heterozygosity $(\mathrm{LOH})$ analysis restricted to APC, DCC, and p53 loci (Vortmeyer et al, 1997), a close genetic relation- 
ship was observed between the two components, regardless of the typology of the mixed tumors analyzed. Because additional genetic alterations restricted to PDEC have been detected, it was speculated that a mixed exocrine-endocrine tumor evolves from a single epithelial precursor following an exocrine to endocrine cell-type sequence and not vice versa (Kim et al, 2002). Moreover, it was emphasized that the differentiation into PDEC may occur at a relatively early stage of carcinogenesis as demonstrated by the existence of mixed PDEC with a minimal exocrine component represented by areas of high grade dysplasia or adenoma. Interestingly, because specimens of pure colorectal PDEC without an exocrine component exhibit genetic changes of the adenomaadenocarcinoma sequence (including APC, DCC, and p53 LOH), it was speculated that both mixed and pure PDECs share histogenesis and pathogenetic mechanisms with adenocarcinoma (Vortmeyer et al, 1997).

With regard to WDETs, a few molecular studies have also been performed to elucidate the potential role of the tumor suppressor genes $A P C, D C C$, and p53 (Ramnani et al, 1999; Vortmeyer et al, 1997). Even though the importance of these genes remains to be clarified, such preliminary findings suggest that WDETs do not share cancerogenetic pathways with adenocarcinomas and may represent genetically distinct neoplasms.

In the present study, we performed an allelotyping analysis of one rectal collision tumor formed by a well differentiated endocrine carcinoma (WDEC) and an adenocarcinoma and of five mixed PDECs/WDECadenocarcinomas or adenomas (two of the stomach and three of the colon). We used 35 polymorphic microsatellite markers covering a series of chromosomal regions considered to be critical in both gastrointestinal exocrine and endocrine tumorigenesis. Some of these genetic loci are known to harbor tumor suppressor genes, including APC (5q21), TP53 (17p13.2), DCC and SMAD4 (18q21), ATM (11q22.3), MEN1 (11q13), VHL (3p25-26), FHIT (3p14.2), and SEN6 (6q27). The aim of this work was to analyze the genetic relationships between the microdissected endocrine and exocrine components of the mixed tumors and the collision tumor defining the clonality and the timing of the genetic events during the progression of these neoplasms.

\section{Results}

One collision neoplasm, five mixed endocrine-exocrine tumors, and one adenocarcinoma from six patients were microdissected. A total of 14 tumor foci (including two microspecimens from the endocrine and exocrine components of each mixed or collision tumor, one microspecimen from a synchronous gastric adenocarcinoma, and one microspecimen from high grade dysplasia focus adjacent to the gastric mixed tumor of Patient 1) were analyzed for $\mathrm{LOH}$ at 35 polymorphic microsatellite markers covering chromosomes $3,5 q, 6$, 11, 17, and 18 (Table 1). The detailed results of the microallelotyping analysis are shown in Table 2.
Table 1. Chromosomal Locations of the Microsatellite Marker Set Used for the Allelotyping Analysis

\begin{tabular}{|c|c|c|}
\hline $\begin{array}{c}\text { Chromosome } \\
\text { arm }\end{array}$ & Locus Cytogenetic band & $\begin{array}{l}\text { Known or putative } \\
\text { genes contained }\end{array}$ \\
\hline $3 p$ & $\begin{array}{ll}1038 & 3 p 26.1-p 25.2 \\
1581 & 3 p 21.2-p 14.2 \\
1606 & 3 p 21.31-p 14.2 \\
1295 & 3 p 21.31-p 14.2 \\
1300 & 3 p 21-p 14.2\end{array}$ & $\begin{array}{l}\text { VHL (3p26-p25) } \\
\text { FHIT (3p14.2) }\end{array}$ \\
\hline $3 q$ & $\begin{array}{l}16143 q 25.2-q 26.2 \\
15803 q 27-q 28\end{array}$ & \\
\hline $5 q$ & APC $5 q 21-q 22$ & APC (5q21) \\
\hline $6 p$ & $16106 p 21-p 22$ & \\
\hline $6 q$ & $\left.\begin{array}{rl}268 & 6 q 21-q 22 \\
261 & 6 q 21-q 22 \\
1639 & 6 q 21-q 22 \\
441 & 6 q 25 \\
415 & 6 q 25 \\
193 & 6 q 27 \\
297 & 6 q 27\end{array}\right]$ & SEN6 (6q27) \\
\hline $11 p$ & 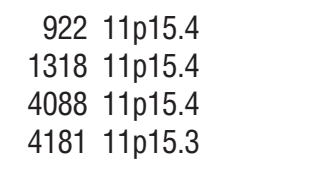 & \\
\hline $11 q$ & 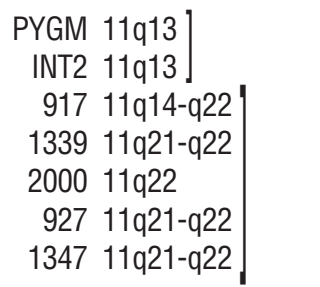 & $\begin{array}{l}\text { MEN1 (11q13) } \\
\text { ATM (11q22.3) }\end{array}$ \\
\hline $17 p$ & TP53 17p13.2 & TP53 (17p13.2) \\
\hline $17 q$ & 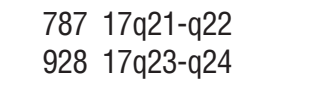 & \\
\hline $18 p$ & $\begin{array}{r}6318 p 11 \\
45218 p 11\end{array}$ & \\
\hline $18 q$ & $\left.\begin{array}{cc}363 & 18 q 21 \\
474 & 18 q 21\end{array}\right]$ & $\begin{array}{r}\text { DCC/SMAD4 } \\
(18 q 21)\end{array}$ \\
\hline
\end{tabular}

Regardless of the primary site, LOH at TP53 locus and/or an immunohistochemical p53 protein accumulation was observed in the endocrine components of all of the five mixed tumors examined (Cases 1 to 5 in Tables 2 and 3). In these cases, the adenocarcinomas associated with the endocrine components invariably shared an identical allelic loss at TP53 marker and/or an immunohistochemical p53 accumulation (Cases 1 to 3 and 5). In the adenoma of the mixed tumor 4 (Table 2), a condition of borderline allelic imbalance at TP53 locus was observed for the same allele lost in the PDEC component. These findings indicating a condition of intratumor heterogeneity for TP53 $\mathrm{LOH}$ were 
Table 2. Results of the Microallelotyping Analysis

\begin{tabular}{|c|c|c|c|c|c|c|c|c|c|c|c|c|c|c|c|}
\hline \multirow{2}{*}{\multicolumn{2}{|c|}{$\frac{\text { Patient No. }}{\begin{array}{c}\text { Hystological } \\
\text { type }\end{array}}$}} & \multicolumn{4}{|c|}{1} & \multicolumn{2}{|c|}{2} & \multicolumn{2}{|c|}{3} & \multicolumn{2}{|c|}{4} & \multicolumn{2}{|c|}{5} & \multicolumn{2}{|c|}{6} \\
\hline & & Adc & D & MuAdc & PDEC & Adc & PDEC & MuAdc & WDEC & $\mathrm{Ad}$ & PDEC & Adc & PDEC & Adc & WDEC \\
\hline \multicolumn{16}{|c|}{$\begin{array}{l}\text { Chromosome } \\
\text { arm and } \\
\text { markers }\end{array}$} \\
\hline \multirow{5}{*}{$3 p$} & 1038 & - & - & - & - & 口 & घ & - & - & $\square$ & $\square$ & nd & $\square$ & $\square$ & a \\
\hline & 1581 & $\square$ & $\square$ & $\square$ & $\square$ & $\Delta$ & $\Delta$ & $\square$ & $\Delta$ & $\square$ & $\square$ & $\square$ & $\square$ & $\square$ & a \\
\hline & 1606 & $\square$ & $\square$ & $\square$ & $\square$ & $\square$ & $\Delta$ & - & - & $\square$ & $\square$ & - & - & nd & nd \\
\hline & 1295 & $\square$ & $\square$ & $\square$ & $\square$ & - & - & nd & nd & - & - & nd & nd & - & - \\
\hline & 1300 & - & - & - & - & nd & nd & $\square$ & $\Delta$ & $\square$ & $\square$ & nd & nd & - & - \\
\hline \multirow{2}{*}{$3 q$} & 1614 & nd & nd & nd & nd & - & - & nd & a & - & - & - & - & $\square$ & $\square$ \\
\hline & 1580 & $\square$ & $\square$ & $\square$ & $\square$ & $\square$ & $\square$ & - & - & nd & nd & nd & nd & - & - \\
\hline $5 q$ & APC & घ & $\square$ & $\square$ & $\Delta$ & $\square$ & $\Delta$ & घ & घ & $\square$ & - & $\Delta$ & $\Delta$ & घ & $\square$ \\
\hline $6 p$ & 1610 & - & - & - & - & $\square$ & $\square$ & - & - & nd & nd & nd & nd & nd & nd \\
\hline \multirow{7}{*}{$6 q$} & 268 & a & $\square$ & $\square$ & $\square$ & $\square$ & $\square$ & $\Delta$ & $\Delta$ & $\square$ & $\square$ & $\square$ & $\square$ & - & - \\
\hline & 261 & $\boldsymbol{\Delta}$ & $\square$ & $\square$ & $\square$ & $\square$ & $\Delta$ & - & - & $\square$ & $\square$ & $\square$ & $\square$ & $\square$ & $\square$ \\
\hline & 1639 & $\Delta$ & $\square$ & $\square$ & $\square$ & $\square$ & घ & $\square$ & $\square$ & $\square$ & $\square$ & $\square$ & $\square$ & - & - \\
\hline & 441 & $\Delta$ & $\boldsymbol{\Delta}$ & $\square$ & $\square$ & $\square$ & $\Delta$ & $\square$ & घ & $\square$ & $\square$ & $\square$ & $\square$ & $\square$ & $\square$ \\
\hline & 415 & a & घ & $\square$ & $\square$ & $\square$ & $\Delta$ & $\square$ & $\square$ & - & - & $\square$ & $\square$ & $\square$ & $\square$ \\
\hline & 193 & $\Delta$ & $\boldsymbol{\Delta}$ & $\square$ & $\square$ & $\square$ & $\Delta$ & $\square$ & $\square$ & - & - & - & - & $\square$ & $\square$ \\
\hline & 297 & - & - & - & - & - & - & - & - & $\square$ & $\square$ & - & - & $\square$ & $\square$ \\
\hline \multirow{4}{*}{$11 p$} & 922 & घ & . & $\square$ & $\square$ & $\square$ & $\square$ & $\square$ & $\square$ & - & - & $\Delta$ & $\Delta$ & $\square$ & $\square$ \\
\hline & 1318 & ! & घ & घ & $\square$ & $\square$ & $\Delta$ & $\square$ & $\square$ & $\square$ & & घ & - & $\square$ & घ \\
\hline & 4088 & $\Delta$ & $\boldsymbol{\Delta}$ & $\square$ & $\square$ & nd & nd & - & - & - & - & - & - & - & - \\
\hline & 4181 & $\bar{\Delta}$ & $\Delta$ & $\square$ & $\square$ & - & - & nd & nd & $\square$ & $\square$ & nd & nd & nd & nd \\
\hline \multirow{7}{*}{$11 q$} & PYGM & a & घ & $\square$ & $\square$ & nd & nd & $\square$ & $\square$ & $\square$ & $\square$ & $\Delta$ & घ & $\square$ & $\square$ \\
\hline & INT2 & ! & घ & $\square$ & $\square$ & घ & घ & $\square$ & $\square$ & $\square$ & $\square$ & $\square$ & घ & $\square$ & $\square$ \\
\hline & 917 & - & - & - & - & $\Delta$ & $\Delta$ & $\square$ & $\square$ & - & - & $\Delta$ & घ & $\square$ & $\Delta$ \\
\hline & 1339 & $\Delta$ & $\Delta$ & $\Delta$ & $\square$ & - & - & - & - & - & - & $\Delta$ & घ & $\square$ & घ \\
\hline & 2000 & घ & घ & घ & $\square$ & nd & nd & nd & nd & nd & nd & nd & nd & - & - \\
\hline & 927 & $\Delta$ & $\boldsymbol{\Delta}$ & $\Delta$ & $\square$ & घ & घ & nd & nd & $\square$ & $\square$ & nd & nd & nd & nd \\
\hline & 1347 & घ & घ & $\square$ & $\square$ & 口 & घ & - & - & - & - & - & - & $\square$ & $\Delta$ \\
\hline $17 p$ & TP53 & $\square$ & घ & $\square$ & $\square$ & $\Delta$ & $\Delta$ & $\square$ & $\square$ & * & $\Delta$ & - & - & घ & $\square$ \\
\hline \multirow{2}{*}{$17 q$} & 787 & - & - & - & - & - & - & $\square$ & $\square$ & $\square$ & $\square$ & - & - & nd & nd \\
\hline & 928 & $\square$ & घ & घ & घ & घ & घ & nd & nd & - & - & 口 & घ & $\boldsymbol{\Delta}$ & $\square$ \\
\hline \multirow{2}{*}{$18 p$} & 63 & nd & nd & nd & nd & - & - & nd & nd & $\square$ & $\square$ & - & - & - & - \\
\hline & 452 & - & - & - & - & $\square$ & $\square$ & $\square$ & $\square$ & nd & nd & nd & nd & $\square$ & $\square$ \\
\hline \multirow{3}{*}{$18 q$} & 363 & $\square$ & $\Delta$ & $\Delta$ & $\Delta$ & - & - & $\Delta$ & $\Delta$ & $\Delta$ & $\Delta$ & $\square$ & $\square$ & - & - \\
\hline & 474 & - & - & - & - & $\square$ & $\Delta$ & - & - & - & - & $\square$ & $\square$ & $\Delta$ & $\square$ \\
\hline & 70 & nd & nd & nd & nd & $\square$ & $\square$ & - & - & $\square$ & $\square$ & $\square$ & $\square$ & घ & $\square$ \\
\hline
\end{tabular}

Adc, adenocarcinoma; D, displasia; MuAdc, mucinous Adc associated with the poorly differentiated endocrine carcinoma; PDEC, poorly differentiated endocrine carcinoma; Ad, adenoma; WDEC, well-differentiated endocrine carcinoma; $\boldsymbol{\Delta}$, LOH of the larger allele; $\mathbf{\square}$, LOH of the smaller allele; $\square$, allelic retention; - , not informative; nd, not determined; *, borderline allelic imbalance of the larger allele.

consistent with a focal immunohistochemical p53 protein accumulation observed in the colorectal adenoma (Table 3).

In addition to TP53 LOH or p53 immunohistochemical abnormalities, D17S928 LOH was also observed in both the components of the tumors 1,2, and 5 . Interestingly, the high grade dysplasia adjacent to the gastric mixed tumor of Patient 1 exhibited loss of the same allele at TP53 and D17S928 locus as the two components of the mixed tumor. On the contrary, the synchronous adenocarcinoma of the same patient showed an allelic retention at the same locus (Fig. 1).

Additional chromosomal regions with concurrent allelic losses in the endocrine and exocrine compo- 
Table 3. Clinicopathological Data of Mixed and Collision Endocrine-Exocrine Tumors

\begin{tabular}{|c|c|c|c|c|c|c|}
\hline Case & Age & Sex & Tumor location & Tumor histology & p53-IR & $\begin{array}{l}\text { Positive immunostainings of the } \\
\text { endocrine components }\end{array}$ \\
\hline \multirow[t]{3}{*}{1} & 62 & M & Stomach & Composite & & \\
\hline & & & & MuAdc & + & \\
\hline & & & & PDEC & + & Syn, NSE, 5HT, VMAT1 \\
\hline \multirow[t]{3}{*}{2} & 37 & M & Stomach & Composite & & \\
\hline & & & & Adc & + & \\
\hline & & & & PDEC & - & Syn, NSE, ChromA \\
\hline \multirow[t]{3}{*}{3} & 52 & $\mathrm{~F}$ & Transverse colon & Combined & & \\
\hline & & & & MuAdc & Rare cells & \\
\hline & & & & WDEC & - & Syn,, NSE, ChromA, 5HT \\
\hline \multirow[t]{4}{*}{4} & 60 & M & Descending & Composite & & \\
\hline & & & colon & $\mathrm{Ad}$ & & \\
\hline & & & & PDEC & focal & \\
\hline & & & & & + & Syn, NSE \\
\hline \multirow[t]{3}{*}{5} & 77 & $\mathrm{~F}$ & Transverse colon & Combined & & \\
\hline & & & & Adc & + & \\
\hline & & & & PDEC & + & Syn, NSE \\
\hline \multirow[t]{3}{*}{6} & 70 & $\mathrm{~F}$ & Rectum & Collision & & \\
\hline & & & & Adc & + & \\
\hline & & & & WDEC & - & Syn, NSE, ChromA, PP, glic \\
\hline
\end{tabular}

IR, immunoreactivity; MuAdc, mucinous adenocarcinoma; PDEC, poorly differentiated endocrine carcinoma; Adc, adenocarcinoma; Ad, adenoma; WDEC well-differentiated endocrine carcinoma; syn, synaptophysin; 5HT, serotonin; VMAT1, vesicular monoamine transporter 1; ChromA, Chromogranin A; PP, pancreatic polypeptide; glic, glicentin; NSE, neuron-specific enolase.

nents were also observed. In particular, $\mathrm{LOH}$ at $18 \mathrm{q}$ markers (including D18S363 and D18S474) was detected in all but one endocrine components of mixed cases examined (Cases 1 to 4), and the same allelic losses were exhibited by the associated adenoma or adenocarcinoma of one gastric (Case 1) and two colorectal mixed tumors (Cases 3 and 4). Analogously, a concurrent loss of the same allele in both components was observed in Case 2 at $11 q$ and $3 p$ loci and in Case 3 at D6S268, respectively. Interestingly, in all colorectal mixed tumors (Cases 3 to 5), the loss of the larger or the smaller allele at APC marker was shared by the associated adenoma or adenocarcinoma.

It is worth noting that in all of the common $\mathrm{LOH}$ regions reported above, a condition of stronger genetic homogeneity (see "Material and Methods" section) was invariably observed in the endocrine than in the exocrine component.

The PDEC and WDEC of three mixed tumors ( $\mathrm{Pa}$ tients 1 to 3 ) exhibited further $\mathrm{LOH}$ regions in addition to those shared with the exocrine components (Table 2). These allelic losses involved 11p, 6q, and 18q arms (Case 2), APC marker (Cases 1 and 2), and chromosome 3 (Case 3).

No additional $\mathrm{LOH}$ regions restricted to the exocrine component were found, with the exception of $\mathrm{LOH}$ at 11 chromosome markers (D11S1339, D11S2000, D11S927, and D11S1318) observed in only the adenocarcinoma of the mixed tumor of Patient 1 . In one specimen (Case 5), allelic loss of the opposite allele at 11q markers (PYGM, D11S917, and D11S1339) was observed in the PDEC component compared with the adenocarcinoma.
Comparing the genetic similarities among the four microdissected components from the two tumors of Patient 1 (Adc, D, MuAdc, and PDEC/WDEC in Table 2) only one common LOH region at 11q22 (D11S927, D11S2000, and D11S1339) was shared by both the adenocarcinomas (Adc and MuAdc microspecimens) together with the high grade dysplasia ( $D$ microspecimen). On the contrary, the PDEC component from the mixed tumor showed no common $\mathrm{LOH}$ region with the synchronous adenocarcinoma (Fig. 1). Interestingly, a strong genetic relationship was observed comparing the allelotypes of microspecimens $D$ and Adc that shared an extensive loss of all informative markers on chromosome 11 and 6q25-6q27 region (Table 2).

Only one collision tumor composed by a WDEC associated with adenocarcinoma occurred (Case 6), and completely different allelotypes between the two components were observed. In particular, whereas the adenocarcinoma showed LOH at APC and at chromosome 17 and 18q markers (TP53, D17S928, D18S474, and D18S70), the well differentiated endocrine carcinoma exhibited allelic losses at 3p (D3S1038 and D3S1581) and at chromosome 11 loci (D11S1318, D11S917, D11S1339, and D11S1347) (Fig. 2).

\section{Discussion}

The microallelotyping analysis of heterogeneous tumors exhibiting the coexistence in the same slide either of histologically different components or of an early and more advanced stage of the same neoplasm represents one of the most successful approaches to defining the clonality and timing of the genetic events underlying tumor progression at various sites (Abeln et 


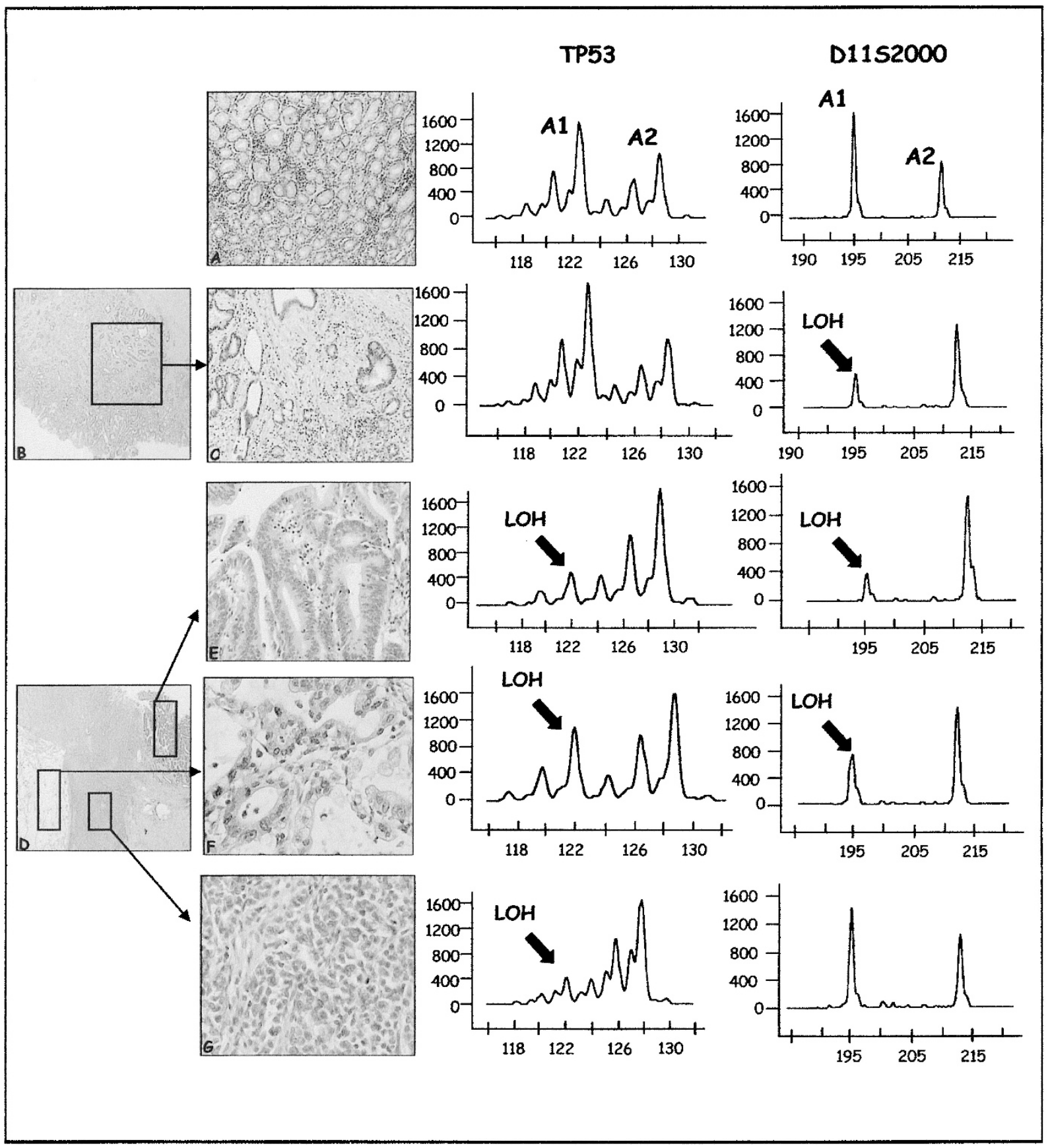

Figure 1.

Allelotypes at TP53 and D11S2000 loci of the five microspecimens from normal gastric mucosa (A) and the two synchronous gastric tumors (B and D) of Patient 1. The electropherograms in the left panel show an identical allelic loss at TP53 locus (arrows) shared by the high grade dysplasia (E), mucinous adenocarcinoma $(\mathrm{F})$, poorly differentiated endocrine carcinoma $(\mathrm{PDEC})(\mathrm{G})$, and allelic retention in the synchronous adenocarcinoma $(\mathrm{B}$ and $\mathrm{C})$. The electropherograms on the right panel show an identical allelic loss at D11S2000 locus (arrows) shared by the synchronous adenocarcinoma (B), high grade dysplasia (E), mucinous adenocarcinoma $(\mathrm{F})$, and allelic retention in PDEC.

al, 1995; Boland et al, 1995; Chen et al, 1992; Macintosh et al, 1998; Shen et al, 2000). The strategy used, which has been successfully applied in defining a model for colorectal tumorigenesis (Fearon and Vogelstein, 1990), is that a prevalent genetic alteration that is consistently seen, regardless of the histologic type, grade, or stage, is considered an early event in the tumorigenesis and supports the hypothesis of a monoclonal origin despite the intratumor heterogene- ity. According to this model, additional genetic alterations restricted to either advanced stages and grades or to specific histologic types are interpreted as later events during the progression of the tumor.

Mixed exocrine-endocrine tumors display a high degree of intratumor heterogeneity in terms of histologic appearance and immunophenotypic features and do not exhibit easily detectable pathologic tracts to define a sequential evolution. Therefore, it is not 


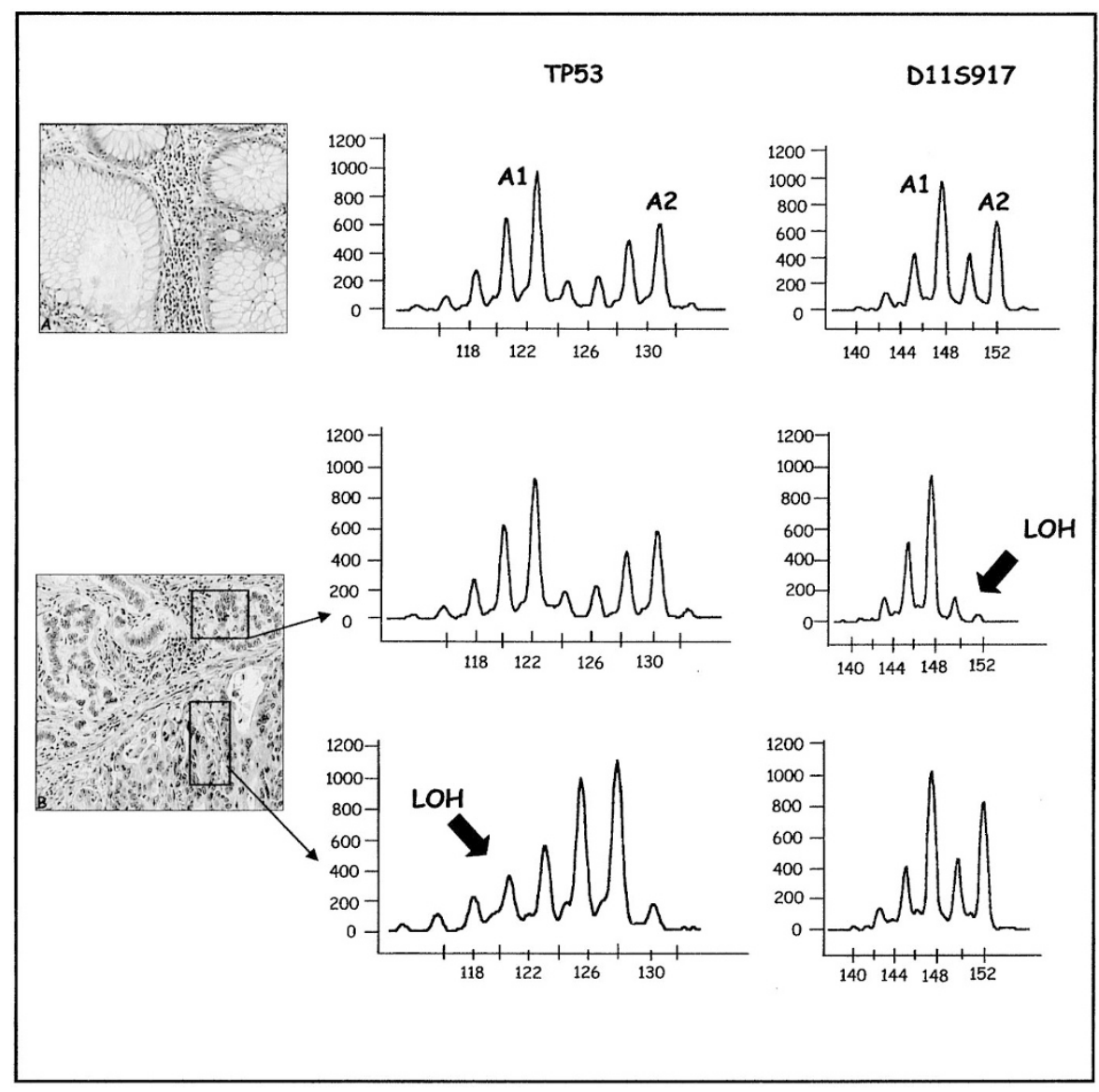

Figure 2.

Allelotypes at TP53 and D11S917 loci of normal tissue (A), WDEC, and adenocarcinoma component (B) from the mixed tumor of Patient 6. In the adenocarcinoma component, TP53 LOH (arrow) and allelic retention at D11S917 marker are evident. On the contrary the WDEC component shows D11S917 LOH (arrow) and allelic retention at the TP53 marker.

clear if the biphenotypic differentiation represents a genetic continuum from a common pluripotent stem cell or if the exocrine and endocrine tumor components arise from two distinct precursors through different tumorigenetic pathways (Kim et al, 2002; Peison and Benisch, 1983; Vortmeyer et al, 1997; Yamashita and Flinner, 1985).

In this study, a strong genetic relationship between the exocrine and endocrine components was demonstrated in all mixed tumors examined (Cases 1 to 5 in Table 2) regardless of the primary site, type, or grade of the mixed tumor. Both components of these five cases exhibited primary common genetic alterations and secondary alterations restricted to the endocrine component (Table 2). These results strongly support the hypothesis of a monoclonal origin of the two components from a pluripotent epithelial stem cell that undergoes a biphenotypic differentiation after carcinogenesis is initiated. Therefore, the endocrine component of a mixed carcinoma with juxtaposed areas of high grade dysplasia, adenoma, or adenocarcinoma could represent a differentiation spectrum of the same tumor. Because the PDECs invariably showed a stronger intratumor homogeneity for $\mathrm{LOH}$ than the exocrine component and additional allelic losses restricted to PDECs were often observed, it is conceivable that the endocrine differentiation may be from an exocrine to an endocrine cell type and not vice versa.

In this scenario, the association of PDEC with adenoma (Case 4) provides evidence that the differentiation into small cell carcinoma may occur at a relatively early stage in the gastrointestinal carcinogenesis. Moreover, these results are in agreement with the findings reported in two recent microallelotyping analyses studying seven mixed colorectal PDECs and eight mixed gastric PDECs (Kim et al, 2002; Vortmeyer et al, 1997). Interestingly, Vortmeyer et al reported the presence of genetic changes of the adenoma-adenocarcinoma sequence (including APC, DCC, and p53 LOH) not only in mixed PDECs but also in pure PDECs of the colon-rectum, speculating that both types of tumors share both the histogenesis and the pathogenetic mechanisms with the colorectal adenocarcinoma. In our series, TP53 LOH and/or immunohistochemical p53 protein accumulation was always found in both components of the five mixed tumors examined (Tables 2 and 3). Moreover, considering only the PDEC or WDEC component, APC LOH was found in all five mixed tumor and allelic deletion at $18 \mathrm{q}$ markers in two gastric and two colorectal cases. Notwithstanding the small number of cases examined in our 
study, these findings suggest a recurrent involvement of $5 q, 17 p$, and $18 q$ chromosome arms in all of the endocrine components analyzed, regardless of the primary site. In particular, TP53 LOH or monosomy of chromosome 17 seems to be the common feature of all mixed tumors examined, probably representing a very early genetic event in the development of these tumors. It is intriguing to find that the TP53 gene is directly involved in a molecular pathway related to cellular responses against double-strand breaks (DSBs) arisen in DNA (Kanaar et al, 1998; Venkitaraman, 1999). Given the central role of DSB-induced chromosome breakage to cause genomic deletion or nonhomologous recombination (Moynahan and Jasin, 1997; Windle et al, $1991 ;)$, it is conceivable that both mixed and pure endocrine tumor etiology is specifically linked to a chromosomal instability initiated by DSBs. In agreement with this hypothesis, the allelotypes of the five mixed tumors examined showed evidence of aneuploidy, because an extensive loss of all informative markers on particular chromosomal arms (including $6 q$ and $3 p)$ or even of the whole chromosome (chromosomes 3, 11, and 17) was found (Table 2).

In Patient 1 it was possible to compare the allelotypes of the mixed PDEC-adenocarcinoma (considering all of the three microspecimens of high grade dysplasia, mucinous adenocarcinoma, and PDEC) and the synchronous adenocarcinoma of the stomach (Fig. 1). Interestingly, whereas the PDEC component of the mixed tumor showed no common $\mathrm{LOH}$ region with the synchronous adenocarcinoma, a common genetic LOH at 11q22 (D11S927, D11S2000, and D11S1339) was shared by both the adenocarcinomas (Adc and MuAdc microspecimens) together with the high grade dysplasia (D microspecimen). The intriguing aspect is that the microspecimen of the high grade dysplasia was surrounding the mixed tumor (Fig. 1) located on the posterior wall of the stomach, and it was distant from the second adenocarcinoma located in the angular region of the stomach. The identical allelotypes at chromosome 11 and 6q26-27 markers of the high grade dysplasia and the adenocarcinoma of the angulus suggests that, despite the physical distance between the two microspecimens, common genetic alterations present through the gastric mucosa could precede the initiation of both the synchronous tumors. This idea is consistent with the observation of three small ulcers in both the angular region and in the posterior wall of the stomach at the time of the esophagogastroduodenoscopy.

In addition to the results concerning the allelotypes of the five PDEC/WDEC-adenocarcinoma/adenoma tumors, this study provides for the first time a molecular support to the identification of collision-type tumors as a rare entity within the spectrum of tumors formed by an endocrine and an exocrine component. In particular, we reported two completely different molecular profiles for the two microspecimens from the only collision tumor of our series formed by a WDEC and an adenocarcinoma. In this tumor, the adenocarcinoma component showed the typical ge- netic changes of the adenoma-adenocarcinoma sequence (such as APC, TP53, and 18q LOH), whereas the WDEC exhibited allelic losses at chromosome 11 and $3 p$ loci but allelic retention at APC, TP53, and 18q markers as reported by Vortmeyer et al (Vortmeyer et al, 1997). These molecular findings, together with the interesting observation that the endocrine and exocrine areas of this tumor gave separate metastases to different lymph nodes without mixture of the two components, confirm the existence of double tumors growing next to each other coincidentally but showing different histogenesis and different tumorigenetic pathways.

In conclusion, this work supports the hypothesis that unlike endocrine-exocrine collision tumors, a monoclonal mechanism of tumorigenesis is the most frequent genetic event in mixed endocrine-exocrine tumors of both composite and combined types and, in particular, seems to be a peculiar feature of neoplastic transformation of the mixed tumors with PDECs.

\section{Materials and Methods}

\section{Patients and Samples}

One collision neoplasm and five mixed endocrineexocrine tumors of the gut were selected from the files of the Department of Pathology of the Varese University Hospital. All tumors were surgically resected and immediately fixed in buffered formalin (4\% [lsqb] w/v [rsqb] formaldehyde and $0.05 \mathrm{~mol} / \mathrm{L}$ acetate buffer) for 24 hours, routinely processed and paraffin embedded. Five- $\mu \mathrm{m}$-thick sections were stained with hematoxylin-eosin, alcian blue-periodic acid Schiff, and Grimelius silver impregnation for the histopathologic evaluation. The main clinicopathologic data of patients are summarized in Table 3 . In the five mixed tumors, the endocrine components consisted of a pure PDEC (three cases) or WDEC (one case) or a PDEC with areas of WDEC (one case), which was associated with adenocarcinoma in four cases (two of them were mucinous adenocarcinomas) and with a villous adenoma in one case. The only collision tumor was located in the rectum and consisted of a well differentiated malignant endocrine tumor (WDEC) associated with an adenocarcinoma. In three of the five mixed tumors, the two components were mainly separated, although close to each other (composite tumors). In two cases the endocrine and exocrine components were mainly intermingled (combined tumor). One of the two gastric tumors (Case 2) presented lymph node metastases at the time of surgery. The other case (Case 1), which was an early gastric cancer confined to submucosa, was not associated with metastases. In this case an adenocarcinoma of intestinal type, completely separated from the mixed tumor, was found in the stomach. Among colonic tumors three out of four neoplasms metastasized (Cases 3,4 , and 6) to regional lymph nodes. Interestingly, the rectal collision neoplasm (WDEC-adenocarcinoma) showed metastases to regional lymph nodes. The endocrine and exocrine components gave separate 
metastases to different lymph nodes, and mixed exocrine-endocrine metastases were not detected.

\section{Immunohistochemistry}

Immunohistochemical investigations were performed in all cases to define the immunophenotype of the endocrine components employing the antibodies and antisera (Table 4). Three- $\mu \mathrm{m}$-thick sections were mounted on poly-L-lysine-coated slides and then deparaffinized and hydrated through graded alcohols to water. Endogenous peroxidase activity was removed by dipping sections in 3\% hydrogen peroxide for 10 minutes at room temperature. Thereafter, sections were incubated with specific antibodies (Table 4) for 18 to 20 hours at $4^{\circ} \mathrm{C}$. Then the avidin-biotinperoxidase procedure was performed according to Hsu et al (1981). The peroxidase activity, indicating the presence of the antigen that we were looking for, was finally revealed by $0.03 \% 3,3^{\prime}$ diaminobenzidine, and nuclei were counterstained with Harris hematoxylin. Sections immunostained for glucagon-glicentin and somatostatin were pretreated with $0.003 \%$ subtilisin (protease type XXVII or Nagarse protease; Sigma, St Louis, Missouri) in $0.05 \mathrm{M}$ Tris-buffered saline $7.4 \mathrm{pH}$ at room temperature for 10 minutes. Sections immunostained for VMAT2 were pretreated with $0.05 \%$ trypsin (Sigma) in 0.05 M Tris-buffered saline $7.4 \mathrm{pH}$ at $37^{\circ} \mathrm{C}$ for 20 minutes, while sections immunostained for synaptophysin, NSE, chromogranin A, and p53 were pretreated with citrate buffer $6 \mathrm{pH}$ in a microwave oven at $700 \mathrm{~W}$ for 10 minutes. Specificity controls consisted of absorption of each antibody with 10 to $20 \mathrm{nMol}$ of related antigen, substitution of the primary antibody with nonimmune serum of the same species at the same dilution, and the use of control tissues with or without the pertinent antigens.

\section{Microdissection and DNA Extraction}

Five- $\mu \mathrm{m}$-thick histologic sections of tumor tissues were obtained from paraffin blocks and stained with hematoxylin-eosin. Using these slides as a guide, microspecimens of the exocrine and the endocrine tumors were carefully scraped with a fine scalpel blade from serial unstained sections from the same paraffin blocks. Homogeneous portions of tumor components were selected to obtain $>80 \%$ purity in each cell population. Control tissue for analysis of constitutional DNA included normal mucosa or lymph node tissue. DNA extraction from microdissected cells was performed as previously described (Furlan et al, 2002).

\section{Allelotyping PCR}

Normal and tumor DNA samples were allelotyped for 35 polymorphic microsatellite markers covering a total of six chromosomes, including 3, 5q, 6, 11, 17, and 18 (Table 3). The markers were selected from the Genome database (www.gdb.org) and from the physical map of the International $\mathrm{RH}$ Mapping Consortium (www.ncbi.nlm.nih.gov/genemap99) on the basis of chromosomal location and heterozygosity. Forward primers were synthesized with a fluorescent tag (FAM, HEX, or TET) on the $5^{\prime}$ end and purified using standard HPLC. Fifty ng of DNA was amplified in a $15-\mu l$ reaction solution containing $1.5 \mu$ of $10 \times$ buffer (Roche, Mannheim, Germany), 1.25 to $2.25 \mathrm{~mm} \mathrm{MgCl}_{2}$, $0.3 \mu \mathrm{M}$ primer pairs, $200 \mu \mathrm{M}$ dNTPs, and 2U DNA polymerase (Roche). Amplifications were performed using a 5-minute initial denaturation at $95^{\circ} \mathrm{C}$, followed by 10 cycles of 50 seconds at $94^{\circ} \mathrm{C}, 50$ seconds at $55^{\circ} \mathrm{C}$, and 50 seconds at $72^{\circ} \mathrm{C}$; and by 25 cycles of 30 seconds at $89^{\circ} \mathrm{C}, 30$ seconds at $55^{\circ} \mathrm{C}$, and 30 seconds at $72^{\circ} \mathrm{C}$. The fluorescently labeled PCR products were subjected to electrophoresis on an Applied Biosystems 310 automated DNA sequencer (Applied Biosystems, Milan, Italy), and the fluorescent signals from the different sized alleles were recorded and analyzed using Genescan software (version 2.1) (Applied Biosystems).

\section{Definition of Allelic Loss}

An imbalance factor was defined as the ratio of relative allelic peak height in the tumor DNA to relative allelic

Table 4. Antibodies and Antisera Used in Immunohistochemical Analyses

\begin{tabular}{llll}
\hline \multicolumn{1}{c}{ Antibodies/antisera } & P/M (clone) & Dilution & \multicolumn{1}{c}{ Source } \\
\hline Synaptophysin (Syn) & M (SY38) & $1: 100$ & BioGenex, San Ramon, California \\
Neuron specific enolase (NSE) & M (H14) & $1: 100$ & Dako, Copenhagen, Denmark \\
Chromogranin A (ChromA) & M (PHE5) & $1: 50$ & Enzo Diagnostics, Farmingdale, New York \\
Glicentin-glucagon (Glic) & $\mathrm{P}$ & $1: 2500$ & Milab, Malmo, Sweden \\
Pancreatic polypeptide (PP) & $\mathrm{P}$ & $1: 4000$ & Cambridge Research Biochemicals, Cambridge, \\
& & & United Kingdom \\
Somatostatin (Som) & $\mathrm{M}(\mathrm{YC7})$ & $1: 10$ & Immunochimica Labometrics, Milan, Italy \\
Serotonin (5HT) & $\mathrm{M}(\mathrm{YC5})$ & $1: 50$ & Biogenesis, Bournemouth, United Kingdom \\
C-terminus gastrin-CCK-cerulein & $\mathrm{M}(\mathrm{B} 4)$ & $1: 10000$ & Farmitalia, Milan, Italy \\
Vesicular monoamine transporter 1 (VMAT1) & $\mathrm{P}$ & $1: 2000$ & Fisher Advanced Immunohistochemicals, Trevose, \\
Vesicular monoamine transporter 2 (VMAT2) & $\mathrm{P}$ & $1: 500$ & Chemicon International, Temecula, California \\
p53 & $\mathrm{M}(\mathrm{D} 0-7)$ & $1: 1000$ & Dako, Copenhagen, Denmark \\
\hline
\end{tabular}

$\mathrm{P} / \mathrm{M}$, polyclonal/monoclonal. 
peak height in the corresponding normal DNA. The formula employed for the calculation was T2: T1/N2: $\mathrm{N} 1$, where $\mathrm{T} 1$ and $\mathrm{N} 1$ are the height values for the smaller allele and T2 and N2 are the height values for the larger allele of the tumor and normal samples, respectively. For informative markers LOH was scored when signal reduction for one allele was of $50 \%$ or more (allelic imbalance factor $\leq 0.5$ or $\geq 2$ ).

Random preferential allele amplification was sometimes seen, resulting in artifactual allelic imbalances in the PCR products. This was seen when using DNA from archival sections as opposed to purified highmolecular-weight DNA. However, this artifact could be distinguished from true allelic losses by repeating the experiments several times (at least three times). Allelic losses were assigned only after the experiments were repeated at least three times and if the losses were found consistently in all repeated experiments.

\section{Evaluation of Intratumor Heterogeneity for $\mathrm{LOH}$}

Because pure tumor-cell contents were used in this analysis by microdissection, we carefully evaluated the degree of allelic signal reduction at all markers in which $\mathrm{LOH}$ was scored to assess the presence of an intratumor heterogeneity rather than an intratumor homogeneity for $\mathrm{LOH}$.

A condition of genetic homogeneity was defined when a tumor DNA showed $\mathrm{LOH}$ with at least $80 \%$ of allelic signal reduction compared with normal DNA. On the contrary the presence of an intratumor genetic heterogeneity was assessed when we observed LOH at one locus with an allelic signal reduction ranging from $50 \%$ to $80 \%$ compared with normal DNA. Moreover, a condition of borderline allelic imbalance (with an allelic signal reduction ranging from $40 \%$ to $50 \%$ ) was thoroughly considered in the analysis of high grade dysplasia samples because of the well known clonal heterogeneity reported in this preneoplastic lesion (Boland et al, 1995).

\section{References}

Abeln ECA, Kuipers-Dijkshoorn NJ, Berns EMJJ, HenzenLogmans SC, Fleuren GJ, and Cornelisse CJ (1995). Molecular genetic evidence for unifocal origin of advanced epithelial ovarian cancer and for minor clonal divergence. $\mathrm{Br} \mathrm{J}$ Cancer 72:1330-1336.

Boland CR, Sato J, Appelman HD, Bresalier RS, and Feinberg AP (1995). Microallelotyping defines the sequence and tempo of allelic losses at tumor suppressor gene loci during colorectal cancer progression. Nat Med 1:902-999.

Capella C, La Rosa S, Uccella S, Billo P, and Cornaggia M (2000). Mixed endocrine-exocrine tumors of the gastrointestinal tract. Sem Diagn Pathol 17:91-103.
Chen L-C, Kurisu W, Ljung B-M, Goldman ES, II DM, and Smith HS (1992). Heterogeneity for allelic loss in human breast cancer. J Nat Cancer Inst 84:506-510.

Fearon ER and Vogelstein BA (1990). A genetic model for colorectal tumorigenesis. Cell 61:759-767.

Furlan D, Casati B, Cerutti R, Facco C, Terraciano L, Capella C, and Chiaravalli AM (2002). Genetic progression in sporadic endometrial and gastrointestinal cancers with microsatellite instability. J Pathol 197:603-609.

Hsu SM, Raine L, and Fanger H (1981). Use of avidin-biotinperoxidase complex (ABC) in immunoperoxidase techniques. J Histochem Cytochem 25:577-580.

Kanaar R, Hoeijmakers JHJ, and van Gent DC (1998). Molecular mechanisms of DNA double-strand break repair. Trends Cell Biol 8:483-489.

Kim KM, Kim MJ, Cho BK, Choi SW, and Rhyu MG (2002). Genetic evidence for the multi-step progression of mixed glandular-neuroendocrine gastric carcinomas. Virchows Arch 440:85-93.

Macintosh CA, Stower M, Reid N, and Maitland NJ (1998). Precise microdissection of human prostate cancers reveals genotypic heterogeneity. Cancer Res 58:23-28.

Moynahan ME and Jasin M (1997). Loss of heterozygosity induced by a chromosomal double-strand break. Proc Natl Acad Sci USA 94:8988-8993.

Peison B and Benisch B (1983). Simultaneous occurrence of malignant carcinoid and adenocarcinoma of stomach. Arch Pathol Lab Med 107:99-100.

Ramnani DM, Wistuba II, Behrens C, Gazdar AF, Sobin LH, and Albores SJK (1999). Ras and p53 mutations in the pathogenesis of classical and goblet cell carcinoids of the appendix. Cancer 86:14-21.

Shen CY, Yu JC, Lo YL, Kuo CH, Yue CT, Jou YS, Huang CS, Lung JC, and Wu CW (2000). Genome-wide search for loss of heterozygosity using laser capture microdissected tissue of breast carcinoma: An implication for mutator phenotype and breast cancer pathogenesis. Cancer Res 60:3884-3892.

Venkitaraman AR (1999). Breast cancer genes and DNA repair. Science 286:1100-1102.

Vortmeyer AO, Lubensky IA, Merino MJ, Wang CY, Pham T, Furth EE, and Zhuang Z (1997). Concordance of genetic alterations in poorly differentiated colorectal neuroendocrine carcinomas and associated adenocarcinomas. J Nat Cancer Inst 89:1448-1453.

Windle B, Draper BW, Yin YX, O'Gorman S, and Wahl GM (1991). A central role for chromosome breakage in gene amplification, deletion formation, and amplicon integration. Genes Dev 5:160-174.

Yamashita M and Flinner RA (1985). Concurrent occurrence of adenocarcinoma and carcinoid tumor in the stomach: A composite tumor or collision tumors? Am J Clin Pathol 83:233-236. 